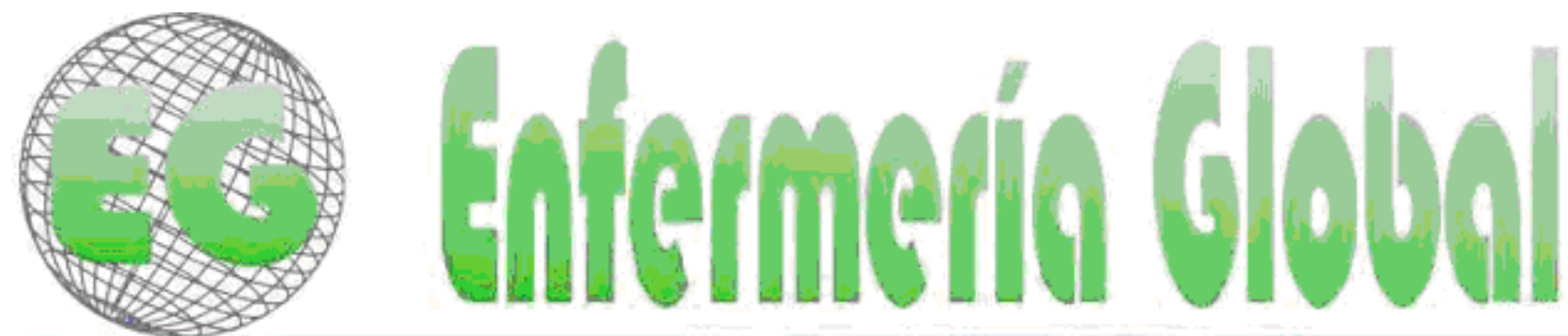

15SN 1696-6*\$S:

Revista electrônica cuatrimestral de Enfermeria

$\mathrm{N}^{\circ} 18$

Febrero 2010

www.um.es/egloball

REFLEXIONES - ENSAYOS

\title{
HOMBRES EN LA ENFERMERÍA PROFESIONAL
}

MEN AT THE NURSING PROFESSION

\author{
${ }^{*}$ Osses-Paredes, C., ${ }^{* *}$ Valenzuela Suazo, S., ${ }^{* *}$ Sanhueza Alvarado, O. \\ *Lcdo. en Enfermería. **Doctora en Enfermería. Docente del Departamento de Enfermería. \\ Universidad de Concepción. Chile
}

Palabras clave: Historia de la enfermería, enfermería, enfermeros.

Keywords: History of Nursing, Nursing, Professional Nursing.

\section{RESUMEN}

Propósito: Analizar las características generales del ingreso de varones a la enfermería, junto con el tendiente incremento de estos en dicha profesión.

Desarrollo: Contrario a la percepción de muchas personas, la enfermería ha sido un campo dominado por los hombres durante la mayor parte de la historia de la humanidad, sólo los hombres eran considerados lo suficientemente "puros" como para convertirse en enfermeros, asociándose esta labor a lo mágico, lo religioso y por otro lado, a lo militar, conociéndose esta etapa como la enfermería no profesional.

Por su parte, la enfermería profesional nace con Florence Nightingale y se asocia al trabajo de la mujer, cuya formación inicial impartida por monjas, rehusaba pensar la consideración de hombres. Así en Chile, en los años sesenta, las carreras de Enfermería eran exclusivas para mujeres. Este suceso se ha hecho sentir, acarreando como consecuencia una subvaloración, y por otro lado, quitándole mérito y reconocimiento a la profesión, por lo que el ingreso masculino ha sido concebido como un hecho positivo para contrarrestar algunas deficiencias, acarreadas por el antiguo sistema biomédico.

En la actualidad existe una tendencia importante de ingreso de hombres, pudiendo referirse a una masculinización de la enfermería, relacionada con las buenas proyecciones laborales, el amplio campo de trabajo, los buenos sueldos y el carácter científico humanista de la profesión.

\section{ABSTRACT}

Purpose: To analyze the general characteristics of the presence of men in nursing history, along with the actual tendency of increase in this profession. 
Development: Contrary to the perceptions of many people, nursing has been a field dominated by men during the majority of human history. Only men were considered "pure" enough to become nurses, this job being associated to magic, religion and also, to the military, this stage being known as nonprofessional nursing.

On the other hand, professional nursing originated with Florence Nigthingale and it has been associated to the jobs of women, whose initial training, given by nuns, refused to acknowledge the considerations of men. Therefore, in the 60s in Chile studying Nursing was exclusively for women. This event is causing as a result an undervaluing of its tasks. Moreover, it has diminished merit and recognition of the profession, which is why the masculine entrance has been perceived as a positive fact that counteracts some deficiencies caused by the old biomedical system.

At present, there exists an important tendency of the entrance of men, making it possible to refer to a nursing masculinization. This phenomenon is related to good career projections, an ample job field, good salaries, and the scientific-humanist professional character of the present era of the Chilean nursing based in care management.

\section{Introducción}

Enfermería es la ciencia del cuidado humano cuya actividad actualmente llevan a cabo las enfermeras y los enfermeros ${ }^{(1)}$, sin embargo en sus inicios había sido una labor especialmente desempeñada por la mujer ${ }^{(2)}$ mientras que las actividades económicas fueron delegadas al trabajo masculino en labores más rentables ${ }^{(2)}$, de control, fuerza y dominio ${ }^{(3)}$.

Inevitablemente al hablar de enfermería profesional, debemos hacer mención a Florence Nightingale, quien a mediados del siglo XIX asiste como voluntaria a la guerra de Crimea y organiza un departamento de enfermería, desafiando los tabúes de la sociedad inglesa. Ella escribe y enuncia una serie de postulados sobre su punto de vista acerca de la enfermería ${ }^{(4)}$, siendo uno de los requisitos para ingresar a su departamento, el ser mujer joven las que debían ser maternales, atentas y $\operatorname{compasivas}^{(5)}$.

En Chile, en los años sesenta las carreras de Enfermería eran exclusivas para mujeres y no se permitía el ingreso de hombres ${ }^{(6)}$. Se consideraba que las labores de este tipo de profesionales era una continuidad de lo doméstico y estaban estrechamente ligadas a lo maternal y a una delicadeza propiamente femenina concordante con el perfil de Florence.

En la actualidad, cada vez ingresan más hombres a la carrera de enfermería, tanto en universidades tradicionales, como en universidades privadas. Este incremento tiene relación con los mejores sueldos y las buenas proyecciones laborales provocando así que la matrícula de enfermeros aumentara en $67 \%$ durante los últimos cinco años ${ }^{(6)}$.

En el presente trabajo, se pretende analizar las características generales del ingreso de hombres a la enfermería junto con el tendiente incremento de estos en dicha profesión.

\section{Feminización de la enfermería profesional}

En 1859, Florence y el Fondo Nightingale comenzaron a negociar la creación de un centro de formación de enfermeras en el hospital St. Thomas. La idea de formar enfermeras no era totalmente nueva en la Gran Bretaña de mediados del siglo XIX. Ya antes de la guerra de Crimea habían comenzado a resurgir las asociaciones de enfermeras, que agrupaban a numerosas mujeres competentes y moralmente intachables, en contraposición al estereotipo de enfermera "borracha e ignorante" creado por Charles Dickens ${ }^{(7)}$, A diferencia de las anteriores, la escuela fundada por Florence, dio paso a la enfermería profesional propiamente como tal. 
Sin embargo, el candil de Nightingale no dio luz inmediatamente; la reforma llegó de manera lenta y penosa, de forma que lo que se conoció como el sistema Nightingale no fue el proyecto ideal imaginado por Florence ${ }^{(8)}$.

Como se puede apreciar, la historia de la enfermería profesional se asocia al trabajo de la mujer. Además, la formación inicial impartida por monjas, rehusaba pensar la consideración de los hombres para el desempeño de dicha profesión. Por un lado la misma sociedad feminizó la profesión y por otro, su fundadora. Asociado a este hecho, se pensaba que no sería un área de interés para los hombres, pues no poseía el reconocimiento por parte de la sociedad patriarcal imperante en el siglo XIX.

La enfermería nace como profesión conformada exclusivamente por mujeres. Según estimaciones de la famosa feminista Gloria Steinem, una profesión se valora menos cuando tiene aproximadamente una tercera parte de mujeres ${ }^{(9)}$. Sin ir más lejos, en la actualidad, 8 de cada 10 profesionales de enfermería son mujeres ${ }^{(10)}$, lo que se ha hecho sentir en la profesión, acarreando como consecuencia una subvaloración, y por otro lado, quitándole mérito y reconocimiento,

Así, numerosas enfermeras atribuyen esta subvaloración al sistema de salud imperante, eminentemente biomédico y a la labor asistencial entre otras, pero la división del trabajo no se asienta en criterios absolutamente técnicos, sino que mantiene una fuerte carga cultural en forma de roles e imágenes de origen doméstico que asignan al rol de cuidados, propio de enfermería, categorías de sumisión, inferioridad y dependencia ${ }^{(11)}$.

Cabe hacer mención, que no ha sido únicamente el hecho de ser la enfermería un colectivo de mujeres en su mayoría, la causa de su falta de autonomía y de reconocimiento, sino que diversas y complejas cuestiones han tenido importancia además de ser mujeres. El propio desarrollo histórico de la profesión, e incluso la relación con la medicina" ${ }^{(12)}$.

Cabe señalar que el apogeo del desarrollo de la enfermería, reflejado por la creación de teorías científicas del cuidado profesional, ocurrió en forma posterior al inicio del movimiento de liberación femenina en la segunda mitad del siglo XX, siendo este movimiento feminista impulsador del desarrollo del cuerpo de conocimiento de la enfermería ${ }^{(13)}$, este hecho influye aún más en la escasa consideración del hombre para dicha actividad y ayuda a comprender el porqué de la feminización.

Otro hecho que influyó negativamente en el desarrollo de la enfermería profesional, especialmente a nivel mundial, fue el hecho de las mujeres haber cedido su cuerpo de conocimientos y su experticia como "sanadoras", y luego como "parteras" a favor de los hombres y el haber dejado de ser "cuidadoras" para convertirse en "auxiliares de los médicos" dentro de la organización institucionalizada de atención a los enfermos ${ }^{(14)}$.

Entonces, ¿por qué a pesar de todas estas dificultades, los hombres se interesan por esta profesión y poco a poco van provocando la masculinización de la enfermería?

\section{Interés masculino por la Profesión}

Contrario a la percepción de muchas personas, la enfermería y aún más la medicina han sido un campo dominado por los hombres durante la mayor parte de la historia de la humanidad $^{(15)}$. En Atenas, 300 años a.C. existía una ley que prohibía a toda la mujer el 
ejercicio de la medicina, la obstetricia y las tareas relacionadas ${ }^{(16)}$, siendo las ciencias de la salud territorio netamente masculino.

Se habla, que la primera escuela de enfermería en el mundo se inició en la India, alrededor de 250 años a.C. ${ }^{(15)}$ Sólo los hombres eran considerados lo suficientemente "puros" como para convertirse en enfermeros, asociándose esta labor a lo mágico y lo religioso y por otro lado, a lo militar, dadas las numerosas guerras y batallas a lo largo de la historia.

Así, en cada peste que azotó a Europa, los hombres arriesgaron su vida para otorgar cuidados de enfermería, los cuales se encontraban implícitos y probablemente no eran reconocidos como tal. Concretamente, un grupo de hombres, el Parabolani, 300 años d.C inauguraron un hospital, en donde brindaron servicios de cuidado durante la epidemia de la Muerte Negra. Muchos años después, la orden Benedictina de enfermería, "The Alexian Brothers", realizó una tarea similar ${ }^{(15)}$. Todas estas actividades catalogadas como enfermería no profesional, puesto que como se mencionó anteriormente esta recién surge en el siglo XIX.

En la actualidad, cada vez son más los hombres que deciden estudiar enfermería, no precisamente asociado a la religión, sino a la amplia gama de desempeño, las buenas proyecciones laborales y los mejores sueldos ${ }^{(6)}$, lo que se demuestra al momento de realizar las postulaciones para el ingreso a las universidades tradicionales, siendo enfermería una de sus primeras opciones. Por otro lado, y aún más decisorio es el caso del ingreso de los hombres a las universidades privadas, donde el ingreso es plenamente espontáneo, marcado por el interés propio de cada futuro profesional. Con este hecho, se deja de lado el perfil de enfermero que entra a estudiar enfermería por no tener otra opción de ingreso a otra profesión.

Según Futuro Laboral, una institución chilena que mide la proyección laboral en Chile, hoy Enfermería es una carrera en la que existen ciento por ciento de probabilidades de encontrar trabajo al primer año de egreso, lo que la ubica por sobre algunas Ingenierías ${ }^{(6)}$, carreras en que tradicionalmente existe una supremacía masculina, que se contrapone totalmente a enfermería, estimándose para enfermería un sueldo promedio que supera los U\$1.000.

\section{Ingreso masculino a las aulas de enfermería}

El ingreso de hombres, ha generado una restructuración del pensamiento enfermero tanto en los profesionales como en los docentes, e inclusive en el equipo de profesionales y los usuarios. Para suerte de los hombres, han sido bien acogidos por la mayoría de estos, gozando de algunos beneficios al ser minoría.

En las mismas aulas, se diferencia la presencia masculina, siendo más fácil para los docentes aprenderse sus nombres, pasando a ser muy queridos por la clase, por decirlo de alguna manera.

Aun así, la integración no ha sido del todo, al dirigirse al aula los docentes aún dicen "cuando sean enfermeras", o "nosotras las enfermeras", lo que ha provocado resignación y un tanto de acostumbramiento por parte de los estudiantes, y por otro lado identificación con el término "enfermera". Esto también ocurre a nivel de usuario, que para referirse al profesional de enfermería, lo hace diciendo la "enfermera", siendo que orgullosamente somos enfermeras y enfermeros ambos con las mismas capacidades y herramientas para el actuar. 


\section{Impacto de la masculinización en el desempeño laboral.}

La relación médico-enfermera ha respetado históricamente la misma asignación de roles de género que la prescrita socialmente para las relaciones hombre-mujer, en donde los médicos, que eran hombres tenían el control, la autoridad, el conocimiento, y las enfermeras, que eran mujeres, tenían que acatar órdenes, no tenían ni moral ni científica. Evidentemente este hecho ha cambiado, y se ha visto alterada en cierta medida por la incorporación de las mujeres a la medicina, de los hombres a la Enfermería y de los deseos de autonomía de esta última ${ }^{(17)}$.

Paradójicamente en algunas ocasiones, cuando se quiere felicitar a un profesional de enfermería, se alude a "deberías ser médico" o "no has pensado en estudiar medicina", es decir se reconocen el buen hacer, pero curiosamente, cuando se sienten orgullosos, se confunden y dicen que eres médico ${ }^{(17)}$, reafirmando la subvaloración de la profesión. Esto, enmarcado bajo el alero del antiguo sistema paternalista y predominantemente machista de la sociedad chilena, el cual dejó plasmado en la mayoría de la población una dependencia en el quehacer de la enfermera, y no tan solo en los usuarios, sino también en algunas enfermeras como tal.

Pero por otro lado, existe la sensación de que la relación médico-enfermero, es distinta a la relación médico-enfermera, siendo la primera más horizontal y acotada a la labor propia de enfermería, ya que no se tiende a generar esta visión maternal o doméstica hacia los enfermeros, o si se hace es en menor grado. Por su parte, las enfermeras se caracterizan por hacer muchas más actividades que las propias de su profesión, como se señala en un estudio, donde la enfermera manifiesta; "Me pueden interrumpir diez veces en una mañana, no me están pidiendo trabajo de enfermería, sino que resuelva cualquier otro problema que los usuarios me demandan. Los chicos enfermeros, siguen siendo médicos y a ellos no les interrumpen"(17). Aludiendo a lo anterior, todavía existe el estigma en los usuarios de que si eres hombre eres "médico" y si eres mujer, eres enfermera.

Pero en los sistemas de evaluación de los puestos de trabajo, en la misma Organización Internacional del trabajo se ha visto su parcialidad en cuanto al género ${ }^{(18)}$. Así la feminización de la enfermería repercute en la distribución de los puestos de trabajo ${ }^{(5)}$ pues los enfermeros se desenvuelven preponderantemente en servicios de Traumatología, Salud mental, Servicios de emergencias y SAMU, en cambio las mujeres eligen Pediatría ${ }^{(6)}$. Por otro lado cabe señalar que el $6 \%$ de los afiliados en el Colegio de Enfermeras chileno, son varones $^{(6)}$.

Así los hombres tienden a hacer notar sus características masculinas a través de un comportamiento impulsado por una ambición y un deseo de logro que es característico de la masculinidad hegemónica ${ }^{(19)}$, este hecho explica en parte el porqué de la diferencia al elegir un lugar de trabajo, en donde los enfermeros tienden a acceder a aquellos puestos dentro de la enfermería que requieren de dichas características, además de considerar en algunas ocasiones su trabajo de enfermeros como un paso intermedio en el logro de otros objetivos $^{(2,19)}$, metas más altas como la búsqueda de cargos de mayor jerarquía; 0 consideran su empleo como algo transitorio para proseguir sus estudios en otras carreras, tales como la medicina y la administración hospitalaria ${ }^{(19)}$ para gozar de un mayor reconocimiento social.

En contraposición las enfermeras, que por su parte generalmente ven su trabajo como algo permanente, y de cierta forma autolimitan su futuro por el hecho de que la mayoría tiene como objetivo fundar una familia y tener hijos ${ }^{(20)}$. 


\section{CONSIDERACIONES}

La enfermería existente previa a Florence, es decir la enfermería no profesional, fue predominantemente masculina en sus inicios ejercida por un llamado divino, una necesidad o asociado a lo cultural. Luego con la profesionalización pasó a ser una actividad exclusivamente femenina.

En la actualidad existe una tendencia importante de ingreso de hombres a dicha carrera, pudiendo referirse a una masculinización de la enfermería, en cierta medida relacionada con las buenas proyecciones laborales, el amplio campo de trabajo, los buenos sueldos y el carácter científico humanista de la profesión.

Esta tendencia de los hombres a desempeñar trabajos no tradicionales, como es el caso de la enfermería junto con los educadores y trabajadores sociales, tienden a hacer énfasis en aquellas conductas y actitudes que los diferencian de las mujeres ${ }^{(19)}$. Por ejemplo, la inconformidad mostrada por la permanencia en un mismo nivel o puesto y la búsqueda de una mejor posición social, evidenciada en la prosecución de estudios.

Por otro lado, muchas enfermeras y enfermeros sienten que los cuidados que prestan, son eficientes y poco reconocidos, es decir, faltos de valor. Y su hipótesis para explicar esto, reside en el hecho de ser mayoritariamente mujeres, por lo que la masculinización de la enfermería es concebido con un hecho positivo para un mayor reconocimiento, para mejorar las condiciones de trabajo, para la consideración en el aumento de la planta de personal, para quitar el freno al desarrollo académico, aumentar los salarios, entre otros, ya que los que gobiernan, legislan y dirigen en su mayoría son hombres, hecho determinante.

En la era de la enfermería actual, en Chile, la "Gestión del Cuidado", según lo dispuesto en la norma general administrativa №19, otorga la atribución para organizar, supervisar, evaluar y promover el mejoramiento de la calidad de los cuidados de enfermería ${ }^{(21)}$, hecho que hará aún más atractivo el ingreso a la carrera, tanto de hombres como mujeres asegurando la mantención del legado enfermero por varios años más, asociado a las buenas proyección en el ámbito académico y laboral para la profesión.

Por tanto creemos, es necesario reunir las virtudes femeninas y masculinas, haciendo de estas un complemento para el desarrollo pleno de la profesión, logrando así el reconocimiento, la eficiencia y la valoración que nuestros antepasados pretendieron y que nosotros debemos cosechar, Ya que la enfermería más que ser desempeñada por hombres o mujeres, debe ser desempeñada por profesionales de enfermería, es decir enfermeros y enfermeras en su conjunto.

\section{BIBLIOGRAFÍA}

1. Concha G. Género y enfermería. Index Enferm. 2004; 13(46): 07-08.

2. Hernández A. La masculinidad y los empleos no tradicionales, El caso de los enfermeros. La ventana, 1997: 6.

3. Piqué Prado E. Mujer y salud desde una perspectiva de género. Metas Enferm, 2003; 53: 50-54.

4. Hernandez A, Guajardo C. La enfermería como disciplina profesional holística. Rev Cubana Enfermer 2004; 20(2)

5. Celma M, Acuña A. Influencia de la feminización de la enfermería en su desarrollo profesional. Rev. Antropología Experimental. 2009; 9 (9): 119-136 
6. Villablanca M, Valdivieso F, Pavez K. Numero de hombres creció $67 \%$ en cinco años. Archivo de prensa LUN, Enero 2009.

7. Attewell A. Florence Nightingale. Rev trimestral de educación comparada UNESCO. 1998; 28 (1): 173-189

8. Baly, M.E. Florence Nightingale and the nursing legacy. Rieno unido 1986. pag 230, comentado en Attewell A. 1998. Rev trimestral de educación Unesco 28 (1): 173-189

9. Consejo Internacional de Enfermeras (CIE). La enfermería importa, articulo Igualdad de oportunidades: Problemas de género 2009. Disponible en

10. Sixth Meeting of the World Health Organization's (WHO) multi-disciplinary Global Advisory Group for Nursing and Midwifery. 2000 comentado en Fajardo ME, Concha G. Index Enferm. 2004 ; 13(46): 09-12.

11. Alberdi R. 1995 Las enfermeras como trabajadoras de empresas en crisis. Motivación y desarrollo profesional. Rev Rol enf. 198: 13-16.

12. Domñinguez C. Feminización de las profesiones sanitarias 1990, en Jano, (38): 69-74. Comentado en: Revista de Antropología Experimental, 2009; 9 (9): 119-136.

13. Urra E. La teoría feminista post-estructuralista y su utilidad en la ciencia de enfermería. Cienc. enferm. 2007; 13(2): 9-16.

14. Velandia A. Futuro e identidad de enfermería con base en el cuidado, una historia prospectiva del cuidado de enfermería en Colombia. Dimensiones del cuidado. Bogota: Facultad de enfermería de la universidad nacional de Colombia, 1998: 134-151

15. Acevedo A, Marty P, Pacheco S. Clase Hombres en enfermería, Recinto Universitario De Mayagüez disponible en http://www.slideshare.net/enfe3005/hombres-en-enfermeria

16. Alcazas MA. Las mujeres en las ciencias de la salud, DIKAIOSYNE Revista de filosofía práctica Universidad de Los Andes, Venezuela. 2005; 15 (8): 163-174.

17. Fajardo $\mathrm{M}^{\mathrm{a}} \mathrm{E}$, Concha $\mathrm{G}$. Influencia del género en el reconocimiento de los cuidados enfermeros visibles e invisibles. Index Enferm. 2004; 13(46): 09-12.

18. Organización Internacional del Trabajo. Igualdad de oportunidades y trato entre hombres y mujeres en los servicios de salud y médicos. Ginebra: OIT, 1992.

19. Williams C. Estill a Man's World, University of California Press, Berkeley, 1995. Comentado en Hernández A. La ventana 1997. 6.

20. Gilles Lipovestsky. El poder de la eterna diferencia. En público, Guadalajara, 27 de abril de 1998, p2 comentado en Hernández A. La ventana, 1997: 6.

21. Norma General Administrativa No 19 "Gestión del cuidado de enfermería para la atención cerrada”, Exenta № 1127, del Ministerio de Salud. Santiago, 14 de diciembre de 2007, publicada el 25 de diciembre de 2007. Disponible en:

http://www.colegiodeenfermeras.cl/datos/ftp/norma.doc

ISSN 1695-6141

() COPYRIGHT Servicio de Publicaciones - Universidad de Murcia 